PROCEEDINGS OF THE

AMERICAN MATHEMATICAL SOCIETY

Volume 125, Number 9, September 1997, Pages 2571-2579

S 0002-9939(97)03064-5

\title{
CHARACTERIZATION OF THE DUALS OF LATTICES OF CONTINUOUS FUNCTIONS WITH RESPECT TO DISJOINTNESS PRESERVING GROUPS
}

\author{
ANDREY Y. BIYANOV
}

(Communicated by Palle E. T. Jorgensen)

\begin{abstract}
The duals of $C_{0}(a, b)$ and $C[a, b]$ with respect to disjointness preserving groups are characterized. A. Plessner's result (1929) about the translation group is extended. A Wiener-Young type theorem for disjointness preserving groups is obtained.
\end{abstract}

It is assumed that the reader is familiar with the notions of a Banach lattice, positive operator and disjointness preserving operator. $[\mathrm{AB}],[\mathrm{LZ}]$ and $[\mathrm{MN}]$ are good references for this material. We also assume familiarity with the theory of $C_{0}$-semigroups and groups. $[\mathrm{Pa}]$ and $[\mathrm{Na}]$ should provide all necessary information when needed.

Throughout $-\infty \leq a<b \leq+\infty, X$ is either $(a, b)$ or $[a, b]$, where by $[-\infty, b]$ we mean $(-\infty, b]$, and by $[a,+\infty]$ we mean $[a,+\infty)$.

Definition. A function $f: X \rightarrow \mathbb{C}$ is called vanishing at infinity if $\forall \varepsilon>0 \exists K \subset$ $X, K$ compact such that $\forall x \in X \backslash K|f(x)|<\varepsilon$.

We denote the lattice of all continuous functions $f: X \rightarrow \mathbb{C}$ vanishing at infinity by $C_{0}(X)$, the lattice of all bounded continuous functions $f: X \rightarrow \mathbb{C}$ by $C_{b}(X)$, the lattice of all continuous functions $f: X \rightarrow \mathbb{C}$ with compact support by $C_{c}(X)$, and the lattice of all regular complex-valued Borel measures on $X$ with finite variation by $M(X)$.

Let $\{T(t)\}_{t \in \mathbf{R}}$ be a $C_{0}$-group on a Banach space $E$.

Definition. The group dual of $E$ with respect to $\{T(t)\}_{t \in \mathbf{R}}$, denoted $E^{\odot}$ and pronounced $E$-sun, is defined in the following way:

$$
E^{\odot}=\left\{u^{*} \in E^{*}: \lim _{t \rightarrow 0}\left\|T^{*}(t) u^{*}-u^{*}\right\|=0\right\} .
$$

$[\mathrm{vN}]$ is an excellent source of information about the semigroup and group duals of Banach spaces and related subjects.

Definition. A group $\{T(t)\}_{t \in \mathbf{R}}$ on a Banach lattice $E$ is called disjointness preserving if $\forall t \in \mathbb{R} T(t)$ is a disjointness preserving operator.

Let $\{T(t)\}_{t \in \mathbf{R}}$ be a disjointness preserving $C_{0}$-group on $C_{0}(X)$. It follows from [Na, B-II.3.8] that $\exists \varphi: \mathbb{R} \times X \rightarrow X$ a continuous flow $\exists q: \mathbb{R} \rightarrow C_{b}(X)$ a

Received by the editors September 2, 1994.

1991 Mathematics Subject Classification. Primary 47D03, 46B10, 46E05, 47B65.

Key words and phrases. $C_{0}$-group, disjointness preserving operator, group dual, flow, cocycle. 
continuous cocycle of $\varphi$ such that $\forall t \in \mathbb{R} \forall f \in C_{0}(X) \forall x \in X(T(t) f)(x)=$ $q_{t}(x) f\left(\varphi_{t}(x)\right)$, where $\varphi_{t}=\varphi(t, \cdot), q_{t}=q(t)$.

In the sequel we are going to use the following elementary observation.

Lemma 1. $\forall t \in \mathbb{R}\left\|q_{t}\right\|=\|T(t)\|$.

In [Na, B-II.3.21] W. Arendt characterized all continuous flows on $X$. For the sake of completeness we list this result here providing a somewhat more detailed proof than the original one.

Theorem 2. $\varphi$ is a continuous flow on $X$ if and only if the following conditions are satisfied.

(1) $\exists U \subset X, U$ is the union of pairwise disjoint intervals $\left(a_{i}, b_{i}\right), i \in I$, where $I$ is either finite or countable.

(2) $\exists \psi: U \rightarrow \mathbb{R}$ such that $\forall i \in I \psi_{i}=\left.\psi\right|_{\left(a_{i}, b_{i}\right)}:\left(a_{i}, b_{i}\right) \rightarrow \mathbb{R}$ is a homeomorphism.

(3) $\forall t \in \mathbb{R}$

$$
\varphi_{t}(x)= \begin{cases}\psi_{i}^{-1}\left(\psi_{i}(x)+t\right) & \text { if } x \in\left(a_{i}, b_{i}\right), \\ x & \text { if } x \notin U .\end{cases}
$$

Proof. Sufficiency. First we will establish that $\varphi$ is a flow on $\mathbb{R}$. Let $x, s, t \in \mathbb{R}$. Then either $x \in U$ or $x \notin U$. If $x \notin U$, then $\varphi_{t}(x)=\varphi_{s}(x)=\varphi_{t+s}(x)=x$ and therefore $\varphi_{t}\left(\varphi_{s}(x)\right)=\varphi_{t+s}(x)$. If $x \in\left(a_{i}, b_{i}\right)$ for some $i \in I$, then

$$
\varphi_{t}\left(\varphi_{s}(x)\right)=\psi_{i}^{-1}\left(\psi_{i}\left(\psi_{i}^{-1}\left(\psi_{i}(x)+s\right)\right)+t\right)=\psi_{i}^{-1}\left(\psi_{i}(x)+t+s\right)=\varphi_{t+s}(x) .
$$

It is also clear that $\forall x \in \mathbb{R} \varphi_{0}(x)=x$.

The next step is to prove the continuity of $\varphi$. It is fairly clear that if $x \in U$ or $x \notin \bar{U}$, then $\forall t \in \mathbb{R} \varphi_{t}(x)$ is continuous at $(t, x)$. Suppose $x \in \bar{U} \backslash U, t \in \mathbb{R}$. Since $\varphi_{t}(x)=x$, we have to prove that

$$
\varphi_{s}(y) \rightarrow x \text { as } y \downarrow x, s \rightarrow t \quad \text { and } \quad \varphi_{s}(y) \rightarrow x \text { as } y \uparrow x, s \rightarrow t .
$$

We will prove only the first fact of the above two. The second one can be proved in the same manner.

Let $\varepsilon>0$. Then there are two possibilities: either $(x, x+\varepsilon) \subset U$ or $(x, x+\varepsilon) \not \subset U$. Let us consider the second case first. Then $\exists \delta 0<\delta<\varepsilon$ such that $x+\delta \notin U$. Let $x<y<x+\delta$. If $y \notin U$, then $\forall s \in \mathbb{R} \varphi_{s}(y)=y$ and therefore $x<\varphi_{s}(y)<x+\varepsilon$. If, on the other hand, $y \in\left(a_{i}, b_{i}\right)$ for some $i \in I$, then

$$
x \leq a_{i}<y<b_{i} \leq x+\delta<x+\varepsilon .
$$

Note that $\forall s \in \mathbb{R} \varphi_{s}(y) \in\left(a_{i}, b_{i}\right)$ and therefore $x<\varphi_{s}(y)<x+\varepsilon$.

Assume now $(x, x+\varepsilon) \subset U$. Then $\exists i \in I$ such that $a_{i}=x<x+\varepsilon \leq b_{i}$. Since $\psi_{i}$ is a homeomorphism, without losing generality we may assume that it is a decreasing function. Let

$$
\delta=\psi_{i}^{-1}\left(\psi_{i}(x+\varepsilon)+|t|+1\right)-x>0 .
$$

Thus, $\psi_{i}(x+\delta)=\psi_{i}(x+\varepsilon)+|t|+1$. Therefore, $\forall y \in(x, x+\delta), \forall|s|<|t|+1$

$$
\psi_{i}(y)+s>\psi_{i}(x+\delta)-|t|-1=\psi_{i}(x+\varepsilon) .
$$

It follows that $x<\varphi_{s}(y)<x+\varepsilon$. This concludes the proof that $\varphi_{s}(y) \rightarrow x$ as $y \downarrow x, s \rightarrow t$. 
NeCESSITY. First we will prove that $\forall t \in \mathbb{R} \varphi_{t}$ is a strictly increasing function. Assume this is not true. Then $\exists t \in \mathbb{R} \exists x, y \in X, x<y$ such that $\varphi_{t}(x) \geq \varphi_{t}(y)$. Since $\varphi$ is a continuous function and since $\varphi_{0}=\operatorname{id}_{X}, \exists s \in(0, t]$ such that $\varphi_{s}(x)=$ $\varphi_{s}(y)$. Contradiction with the fact that $\varphi_{s}$ is a homeomorphism.

Let $K=\left\{x \in X: \forall t \in \mathbb{R} \varphi_{t}(x)=x\right\}$. Clearly $K$ is a closed set. Also if $X=[a, b]$, it is easy to see that $a, b \in K$. Therefore, $U=X \backslash K$ is open in $\mathbb{R}$ and thus is a union of of pairwise disjoint intervals $\left(a_{i}, b_{i}\right), i \in I$, where $I$ is either finite or countable.

Let $i \in I, x \in\left(a_{i}, b_{i}\right), \beta(t)=\varphi_{t}(x)$. We claim that $\beta$ is an injection. Suppose this is false, i.e. $\exists r, s \in \mathbb{R}$ such that $\varphi_{s}(x)=\varphi_{r}(x)$ which means that $\varphi_{s-r}(x)=x$. Scaling by $t$, if necessary, we may assume that $\varphi_{1}(x)=x$. Let $y=\varphi_{1 / 2}(x)$. It follows from the definition of a flow that $\varphi_{1 / 2}(y)=x$. Since as we showed above $\varphi_{1 / 2}$ is a strictly increasing function, we conclude that $x=y$.

Using a similar argument, we can show that $\forall n \in \mathbb{N} \varphi_{1 / 2^{n}}(x)=x$ and thus $\forall m \in \mathbb{Z} \varphi_{m / 2^{n}}(x)=x$. Since numbers $\left\{m / 2^{n}\right\}_{n \in \mathbb{N}, m \in \mathbb{Z}}$ are dense in $\mathbb{R}$ and since $\varphi$ is a continuous flow, it follows that $\forall t \in \mathbb{R} \varphi_{t}(x)=x$, i.e. $x \in K$. Contradiction with $x \in\left(a_{i}, b_{i}\right)$. Thus, $\beta$ is an injection.

Our next claim is that $\beta$ maps $\mathbb{R}$ onto $\left(a_{i}, b_{i}\right)$. Suppose $\varphi_{t}(x) \notin\left(a_{i}, b_{i}\right)$. Then $\exists s \in(0, t]$ such that $y=\varphi_{s}(x) \in K$. From the definition of $K$ it follows that $\varphi_{-s}(y)=y$ and thus $x=y \in K$. Contradiction with $x \in\left(a_{i}, b_{i}\right)$. We conclude that the image of $\beta$ is contained in $\left(a_{i}, b_{i}\right)$. Thus, $\beta: \mathbb{R} \rightarrow(c, d)$ is a homeomorphism and $(c, d) \subset\left(a_{i}, b_{i}\right)$. Without loss of generality we may assume that $\beta$ is an increasing function. Therefore, $\beta(t) \rightarrow d$ as $t \rightarrow+\infty$. Let $s \in \mathbb{R}$. Then

$$
\varphi_{s}(d)=\varphi_{s}\left(\lim _{t \rightarrow+\infty} \varphi_{t}(x)\right)=\lim _{t \rightarrow+\infty} \varphi_{s}\left(\varphi_{t}(x)\right)=\lim _{t \rightarrow+\infty} \varphi_{t+s}(x)=d .
$$

Hence, $d \in K$ and thus $d=b_{i}$. Analogously $c=a_{i}$. This establishes the claim.

Finally, let $\psi_{i}=\beta^{-1}, y \in\left(a_{i}, b_{i}\right), s=\psi_{i}(y)$, i.e. $y=\varphi_{s}(x)$. Then

$$
\varphi_{t}(y)=\varphi_{t}\left(\varphi_{s}(x)\right)=\varphi_{t+s}(x)=\psi_{i}^{-1}(s+t)=\psi_{i}^{-1}\left(\psi_{i}(y)+t\right) .
$$

Remark. If $\varphi$ is a continuous flow, then $\forall n \in \mathbb{Z} \varphi_{n}=\varphi_{1}^{n}$, i.e. $\varphi_{n}$ is the $n$th iterant of the function $\varphi_{1}$. Theorem 2 shows that the functions $\left\{\varphi_{t}\right\}_{t \in \mathbf{R}}$ are the continuous iterants of $\varphi_{1}$ in the sense of Abel, i.e. there exists a function $\psi$ such that $\varphi_{1}$ satisfies the Abel equation $\psi\left(\varphi_{1}(x)\right)-\psi(x)=1$ (see [Ku, Ch.VII]), and consequently $\forall t \in \mathbb{R}$ $\forall x \in X \psi\left(\varphi_{t}(x)\right)-\psi(x)=t$.

Suppose $\mu$ is a nonnegative Borel measure on $X, t \in \mathbb{R} . \forall F \subset X, F$ Borel define $\mu_{t}(F)=\mu\left(\varphi_{-t}(F)\right)$. By [DS, III.10.8] $\mu_{t}$ is a nonnegative Borel measure on $X$ and $\forall F \subset X, F$ Borel $\forall f \in C_{0}(X)$

$$
\int_{F} f d \mu_{t}=\int_{\varphi_{-t}(F)} f \circ \varphi_{t} d \mu .
$$

It can also be easily seen that if $\mu \in M(X)$, then so is $\mu_{t}$ and the above equality holds as well.

Suppose $\mu \in M(X), t \in \mathbb{R} . \forall F \subset X, F$ Borel define

$$
\mu_{t}^{\prime}(F)=\int_{F} q_{t} \circ \varphi_{-t} d \mu_{t} .
$$


Since $q_{t} \in C_{b}(X)$, [DS, III.10.4] implies that $\mu_{t}^{\prime} \in M(X)$ and $\forall F \subset X, F$ Borel $\forall f \in C_{0}(X)$

$$
\int_{F} f d \mu_{t}^{\prime}=\int_{F} q_{t} \circ \varphi_{-t} \cdot f d \mu_{t}=\int_{\varphi_{-t}(F)} q_{t} \cdot f \circ \varphi_{t} d \mu .
$$

Lemma 3. $\forall t \in \mathbb{R} \forall \mu \in M(X) \mu_{t}^{\prime}=T^{*}(t) \mu$.

Proof. It follows from above that $\forall t \in \mathbb{R} \mu_{t}^{\prime} \in M(X)$ and $\forall f \in C_{0}(X)$

$$
\int_{X} T(t) f d \mu=\int_{X} q_{t} \cdot f \circ \varphi_{t} d \mu=\int_{X} f d \mu_{t}^{\prime} .
$$

Lemma 4. If $\mu \in M(X)$ and $\left.\mu\right|_{U}=0$, then $\mu \in C_{0}(X)^{\odot}$ with respect to $\{T(t)\}_{t \in \mathbf{R}}$.

Proof. Suppose $t \in \mathbb{R}, F \subset X$ is Borel. Then

$$
\mu_{t}^{\prime}(F)=\int_{X} q_{t} \chi_{\varphi_{-t}(F)} d \mu .
$$

Since $\left.\mu\right|_{U}=0, \mu\left(\varphi_{-t}(F) \cap U\right)=0$ and therefore

$$
\mu_{t}^{\prime}(F)=\int_{X} q_{t} \chi_{\varphi_{-t}(F) \backslash U} d \mu .
$$

Since $\varphi_{-t}$ is a bijection and since $\varphi_{-t}(U)=U, \varphi_{-t}(F) \backslash U=\varphi_{-t}(F \backslash U)$. Also since $\left.\varphi_{-t}\right|_{X \backslash U}=\operatorname{id}_{X \backslash U}, \varphi_{-t}(F \backslash U)=F \backslash U$. We obtain:

$$
\mu_{t}^{\prime}(F)=\int_{X} q_{t} \chi_{F \backslash U} d \mu=\int_{X} q_{t} \chi_{F} d \mu
$$

since $\mu(F \cap U)=0$. Thus,

$$
\mu_{t}^{\prime}(F)-\mu(F)=\int_{X}\left(q_{t}-1\right) \chi_{F} d \mu
$$

Let $\mathcal{F}$ be the set of all partitions $\left\{F_{j}\right\}$ of $X$. Then $\forall t \in \mathbb{R}$

$$
\begin{aligned}
\left\|\mu_{t}^{\prime}-\mu\right\| & =\sup _{\left\{F_{j}\right\} \in \mathcal{F}} \sum_{j=1}^{\infty}\left|\left(\mu_{t}^{\prime}-\mu\right)\left(F_{j}\right)\right| \\
& \leq \sup _{\left\{F_{j}\right\} \in \mathcal{F}} \sum_{j=1}^{\infty} \int_{X}\left|q_{t}-1\right| \chi_{F_{j}} d \mu=\int_{X}\left|q_{t}-1\right| d \mu .
\end{aligned}
$$

Since $\{T(t)\}_{t \in \mathbf{R}}$ is a $C_{0}$-group, it is locally bounded. Hence, by Lemma $1 \exists D>0$ such that $\forall|t| \leq 1\left\|q_{t}\right\| \leq D$ and therefore $\left\|q_{t}-1\right\| \leq D+1$. Since by the definition of a cocycle $q_{0}=1$ and since $(D+1) \chi_{X} \in L^{1}(X, \mu)$, it follows from the Dominated Convergence Theorem that

$$
\int_{X}\left|q_{t}-1\right| d \mu \rightarrow 0 \quad \text { as } \quad t \rightarrow 0
$$

Hence, $\left\|\mu_{t}^{\prime}-\mu\right\| \rightarrow 0$ as $t \rightarrow 0$. Since by Lemma $3 \mu_{t}^{\prime}=T^{*}(t) \mu$, it follows that $\mu \in C_{0}(X)^{\odot}$. 
Lemma 5. Suppose $i \in I, \alpha \in L^{1}\left(\left(a_{i}, b_{i}\right), d \psi\right) . \forall F$ Borel, $F \subset X$ define

$$
\mu(F)=\int_{a_{i}}^{b_{i}} \alpha \chi_{F} d \psi
$$

where $\psi$ is as in Theorem 2. Then $\mu \in C_{0}(X)^{\odot}$ with respect to $\{T(t)\}_{t \in \mathbf{R}}$.

Proof. $d \psi$ is either a nonnegative or nonpositive measure on $\left(a_{i}, b_{i}\right)$. Without loss of generality we may assume it is nonnegative. Suppose $(c, d) \subset\left(a_{i}, b_{i}\right)$. Since $\psi_{i}$ is a continuous function, $d \psi(c, d)=\psi(d)-\psi(c)$. Then $\forall t \in \mathbb{R}$

$$
\begin{gathered}
(d \psi)_{t}(c, d)=d \psi\left(\varphi_{-t}(c), \varphi_{-t}(d)\right)=\psi\left(\varphi_{-t}(d)\right)-\psi\left(\varphi_{-t}(c)\right) \\
=\psi(d)-t-\psi(c)+t=\psi(d)-\psi(c)=d \psi(c, d) .
\end{gathered}
$$

It follows that $\forall G \subset\left(a_{i}, b_{i}\right), G$ open $(d \psi)_{t}(G)=d \psi(G)$. [Ru, 2.18] implies that both $d \psi$ and $(d \psi)_{t}$ are regular. Thus, $\forall F \subset\left(a_{i}, b_{i}\right), F$ Borel $(d \psi)_{t}(F)=d \psi(F)$ which means that $\forall t \in \mathbb{R}(d \psi)_{t}=d \psi$ on $\left(a_{i}, b_{i}\right)$.

Let $F \subset X$ be Borel, $t \in \mathbb{R}$. Then by $(*)$ and $(* *)$

$$
\begin{aligned}
\left(\mu_{t}^{\prime}-\mu\right)(F) & =\int_{a_{i}}^{b_{i}} q_{t} \alpha \chi_{\varphi_{-t}(F)} d \psi-\int_{a_{i}}^{b_{i}} \alpha \chi_{F} d \psi \\
& =\int_{a_{i}}^{b_{i}} q_{t} \circ \varphi_{-t} \cdot \alpha \circ \varphi_{-t} \cdot \chi_{\varphi_{-t}(F)} \circ \varphi_{-t} d \psi_{t}-\int_{a_{i}}^{b_{i}} \alpha \chi_{F} d \psi \\
& =\int_{a_{i}}^{b_{i}}\left(q_{t} \circ \varphi_{-t} \cdot \alpha \circ \varphi_{-t}-\alpha\right) \chi_{F} d \psi
\end{aligned}
$$

since $(d \psi)_{t}=d \psi$ on $\left(a_{i}, b_{i}\right), \chi_{\varphi_{-t}(F)} \circ \varphi_{-t}=\chi_{F}$. Using the argument similar to the one in the proof of Lemma 4 , we conclude that $\forall t \in \mathbb{R}$

$$
\left\|\mu_{t}^{\prime}-\mu\right\| \leq \int_{a_{i}}^{b_{i}}\left|q_{t} \circ \varphi_{-t} \cdot \alpha \circ \varphi_{-t}-\alpha\right| d \psi .
$$

We have shown in the proof of Lemma 4 that $\exists D>1$ such that $\forall|t| \leq 1\left\|q_{t}\right\| \leq D$. Let $\varepsilon>0$. Since $\alpha \in L^{1}\left(\left(a_{i}, b_{i}\right), d \psi\right), \exists g \in C_{c}\left(a_{i}, b_{i}\right)$ such that

$$
\int_{a_{i}}^{b_{i}}|\alpha-g| d \psi<\frac{\varepsilon}{3 D}<\frac{\varepsilon}{3}
$$

Since $(d \psi)_{t}=d \psi$ on $\left(a_{i}, b_{i}\right)$, it follows from $(*)$ that $\forall|t| \leq 1$

$$
\int_{a_{i}}^{b_{i}}\left|q_{t} \circ \varphi_{-t} \cdot \alpha \circ \varphi_{-t}-q_{t} \circ \varphi_{-t} \cdot g \circ \varphi_{-t}\right| d \psi=\int_{a_{i}}^{b_{i}}\left|q_{t} \alpha-q_{t} g\right| d \psi \leq D \frac{\varepsilon}{3 D}=\frac{\varepsilon}{3} .
$$

Let $K=\operatorname{supp} g, K^{\prime}=\varphi(K \times[-1,1]) \subset\left(a_{i}, b_{i}\right)$. Since $\varphi$ is a continuous flow, $K^{\prime}$ is compact. Suppose $x \in\left(a_{i}, b_{i}\right) \backslash K^{\prime}$. It means that $\forall y \in K \forall|t| \leq 1 x \neq \varphi_{-t}(y)$ 
which implies that $\varphi_{t}(x) \neq y$. Hence, $\varphi_{t}(x) \notin K$ and $g\left(\varphi_{t}(x)\right)=0$. It follows that $\forall|t| \leq 1 \operatorname{supp}\left(g \circ \varphi_{t}-g\right) \subset K^{\prime}$. Therefore, $\forall|t| \leq 1$

$$
\left|q_{t} \circ \varphi_{-t} \cdot g \circ \varphi_{-t}-g\right| \leq\|g\|(D+1) \chi_{K^{\prime}} .
$$

Since $\chi_{K^{\prime}} \in L^{1}\left(\left(a_{i}, b_{i}\right), d \psi\right)$, the Dominated Convergence Theorem implies that $\exists \delta>0$ such that $\forall|t|<\delta$

$$
\int_{a_{i}}^{b_{i}}\left|q_{t} \circ \varphi_{-t} \cdot g \circ \varphi_{-t}-g\right| d \psi<\frac{\varepsilon}{3} .
$$

Hence,

$$
\begin{aligned}
& \int_{a_{i}}^{b_{i}}\left|q_{t} \circ \varphi_{-t} \cdot \alpha \circ \varphi_{-t}-\alpha\right| d \psi \\
& \leq \int_{a_{i}}^{b_{i}}\left|q_{t} \circ \varphi_{-t} \cdot \alpha \circ \varphi_{-t}-q_{t} \circ \varphi_{-t} \cdot g \circ \varphi_{-t}\right| d \psi \\
&+ \int_{a_{i}}^{b_{i}}\left|q_{t} \circ \varphi_{-t} \cdot g \circ \varphi_{-t}-g\right| d \psi+\int_{a_{i}}^{b_{i}}|\alpha-g| d \psi \\
&<\frac{\varepsilon}{3}+\frac{\varepsilon}{3}+\frac{\varepsilon}{3}=\varepsilon .
\end{aligned}
$$

Gluing all pieces together, we will obtain that $\forall|t|<\delta\left\|\mu_{t}^{\prime}-\mu\right\|<\varepsilon$. Thus, $\lim _{t \rightarrow 0} \mu_{t}^{\prime}=\mu$ and $\mu \in C_{0}(X)^{\odot}$ with respect to $\{T(t)\}_{t \in \mathbf{R}}$.

Lemma 6. $C_{0}(X)^{\odot} \subset M(X \backslash U) \oplus L^{1}(U, d \psi)$.

Proof. Suppose $\mu \in M(X)$. Then

$$
\mu=\nu+\sum_{i \in I} \mu_{i}
$$

where $\left.\nu\right|_{U}=0$ and $\left.\forall i \in I \mu_{i}\right|_{(X) \backslash\left(a_{i}, b_{i}\right)}=0$. Let $A$ be the infinitesimal generator of $\{T(t)\}_{t \in \mathbf{R}}, \omega_{0}$ the growth bound of $\{T(t)\}_{t \in \mathbf{R}}, \lambda>\omega_{0}$. Since by [vN, 1.3.1] $C_{0}(X)^{\odot}=\overline{D\left(A^{*}\right)}$, it suffices to prove that $R\left(\lambda, A^{*}\right) \nu \in M(X \backslash U)$ and $\forall i \in I$ $R\left(\lambda, A^{*}\right) \mu_{i} \in L^{1}(U, d \psi)$.

Let $f \in C_{0}(X)$. Then it follows from $[\mathrm{Pa}, 1.5 .4]$ that $\forall x \in \mathbb{R}$

$$
(R(\lambda, A) f)(x)=\int_{0}^{+\infty} e^{-\lambda t} q_{t}(x) f\left(\varphi_{t}(x)\right) d t .
$$

Hence, since $\left.\nu\right|_{U}=0$ and $\left.\varphi_{t}\right|_{X \backslash U}=\operatorname{id}_{X \backslash U}$

$$
\begin{aligned}
& \left\langle R\left(\lambda, A^{*}\right) \nu, f\right\rangle=\langle\nu, R(\lambda, A) f\rangle \\
= & \int_{X} d \nu(x) \int_{0}^{+\infty} e^{-\lambda t} q_{t}(x) f(x) d t=\int_{X} f(x) H_{\lambda}(x) d \nu(x),
\end{aligned}
$$


where

$$
H_{\lambda}(x)=\int_{0}^{+\infty} h_{\lambda}(t, x) d t, \quad h_{\lambda}(t, x)=e^{-\lambda t} q_{t}(x) .
$$

Let $\varepsilon=\left(\lambda-\omega_{0}\right) / 2$. Since $\{T(t)\}_{t \in \mathbf{R}}$ is a $C_{0}$-group, it is locally bounded. Hence, by Lemma $1 \exists B>0$ such that $\forall t \in \mathbb{R}\left\|q_{t}\right\| \leq B e^{t\left(\omega_{0}+\varepsilon\right)}$, and $\forall x \in X$

$$
\left|H_{\lambda}(x)\right| \leq \int_{0}^{+\infty}\left|h_{\lambda}(t, x)\right| d t \leq B \int_{0}^{+\infty} e^{-\varepsilon t} d t=\frac{B}{\varepsilon} .
$$

Thus, if $\forall F \subset X, F$ Borel we define

$$
\xi(F)=\int_{F} H_{\lambda}(x) d \nu(x),
$$

then by [DS, III.10.4] $\xi \in M(X)$ and $\xi=R\left(\lambda, A^{*}\right) \nu$. Also since $\left.\nu\right|_{U}=0,\left.\xi\right|_{U}=0$ as well.

Let $i \in I$. Without loss of generality we may assume that $\psi$ is nondecreasing on $\left(a_{i}, b_{i}\right)$. Then

$$
\left\langle R\left(\lambda, A^{*}\right) \mu_{i}, f\right\rangle=\left\langle\mu_{i}, R(\lambda, A) f\right\rangle=\int_{a_{i}}^{b_{i}} d \mu_{i}(x) \int_{0}^{+\infty} e^{-\lambda t} q_{t}(x) f\left(\psi_{i}^{-1}(\psi(x)+t)\right) d t .
$$

Suppose $t=\psi(s)-\psi(x)$. Then by [DS, III.10.8]

$$
\begin{aligned}
& \int_{a_{i}}^{b_{i}} d \mu_{i}(x) \int_{0}^{+\infty} e^{-\lambda t} q_{t}(x) f\left(\psi_{i}^{-1}(\psi(x)+t)\right) d t \\
= & \int_{a_{i}}^{b_{i}} d \mu_{i}(x) \int_{x}^{b_{i}} h_{\lambda}(\psi(s)-\psi(x), x) f(s) d \psi(s) .
\end{aligned}
$$

Applying Fubini's Theorem, we will get:

$$
\begin{aligned}
& \int_{a_{i}}^{b_{i}} d \mu_{i}(x) \int_{x}^{b_{i}} h_{\lambda}(\psi(s)-\psi(x), x) f(s) d \psi(s) \\
= & \int_{a_{i}}^{b_{i}} f(s) d \psi(s) \int_{a_{i}}^{s} h_{\lambda}(\psi(s)-\psi(x), x) d \mu_{i}(x) \\
= & \int_{a_{i}}^{b_{i}} F_{i}(s) f(s) d \psi(s),
\end{aligned}
$$

where

$$
F_{i}(s)=\int_{a_{i}}^{s} h_{\lambda}(\psi(s)-\psi(x), x) d \mu_{i}(x)
$$


We need to show that $F_{i} \in L^{1}\left(\left(a_{i}, b_{i}\right), d \psi\right)$. Again using Fubini's Theorem and [DS, III.10.8], we will obtain:

$$
\begin{aligned}
& \int_{a_{i}}^{b_{i}}\left|F_{i}(s)\right| d \psi(s) \leq \int_{a_{i}}^{b_{i}} d \psi(s) \int_{a_{i}}^{s}\left|h_{\lambda}(\psi(s)-\psi(x), x)\right| d\left|\mu_{i}\right|(x) \\
= & \int_{a_{i}}^{b_{i}} d\left|\mu_{i}\right|(x) \int_{x}^{b_{i}}\left|h_{\lambda}(\psi(s)-\psi(x), x)\right| d \psi(s)=\int_{a_{i}}^{b_{i}} d\left|\mu_{i}\right|(x) \int_{0}^{+\infty}\left|h_{\lambda}(t, x)\right| d t \\
\leq & \frac{B}{\varepsilon}\left|\mu_{i}\right|\left(a_{i}, b_{i}\right)<+\infty
\end{aligned}
$$

since $\forall x \in X \int_{0}^{+\infty}\left|h_{\lambda}(t, x)\right| d t \leq B / \varepsilon$.

Hence, $\forall i \in I R\left(\lambda, A^{*}\right) \mu_{i}=\nu_{i}$, where $\forall F \subset \mathbb{R}, F$ Borel

$$
\nu_{i}(F)=\int_{a_{i}}^{b_{i}} F_{i} \chi_{F} d \psi,
$$

which means that $\nu_{i}$ can be associated with a function from $L^{1}\left(\left(a_{i}, b_{i}\right), d \psi\right)$.

Theorem 7. Let $\{T(t)\}_{t \in \mathbf{R}}$ be a disjointness preserving $C_{0}$-group on $C_{0}(X)$. Then $\exists U \subset X, U$ is the union of pairwise disjoint intervals $\left(a_{i}, b_{i}\right), i \in I$, where $I$ is either finite or countable and $\exists \psi: U \rightarrow \mathbb{R}$ such that $\forall i \in I \psi_{i}=\left.\psi\right|_{\left(a_{i}, b_{i}\right)}:\left(a_{i}, b_{i}\right) \rightarrow \mathbb{R}$ is a homeomorphism and the corresponding group dual $C_{0}(X)^{\odot}=M(X \backslash U) \oplus$ $L^{1}(U, d \psi)$.

Proof. Follows from Lemmas 4, 5 and 6.

Remark. The above theorem generalizes the well-known result of A. Plessner ([Pl]) that if $f: \mathbb{R} \rightarrow \mathbb{C}$ and $\operatorname{Var}_{\mathbf{R}}[f]<+\infty$, then $f$ is absolutely continuous if and only if $\operatorname{Var}_{\mathbf{R}}[f(\cdot+t)-f(\cdot)] \rightarrow 0$ as $t \rightarrow 0$.

The following theorem generalizes the result of N. Wiener and R. C. Young ([WY]) about the behavior of measures on $\mathbb{R}$ under translation. We are going to use the following notation: if $S$ is a subset of a Banach lattice $E$, then $S^{d}$ will denote its disjoint complement in $E$.

Theorem 8. Let $\{T(t)\}_{t \in \mathbf{R}}$ be a disjointness preserving $C_{0}$-group on $C_{0}(X)$. Then $\forall \mu \in M(X)$

$$
\limsup _{t \rightarrow 0}\left\|T^{*}(t) \mu-\mu\right\|=2\left\|\mu_{d}\right\|,
$$

where $\mu_{d}$ is the component of $\mu$ in $C_{0}(X)^{\odot d}$.

Proof. Suppose that $\varphi$ and $q$ are the flow and the cocycle associated with $\{T(t)\}_{t \in \mathbf{R}}$. Then it is not difficult to see that $|T(t)|$ is also disjointness preserving with the flow $\varphi$ and cocycle $|q|$. Thus, by Theorem 7 both groups $\{T(t)\}_{t \in \mathbf{R}}$ and $\{|T(t)|\}_{t \in \mathbf{R}}$ have the same $C_{0}(X)^{\odot}$.

Let $m$ be the Lebesgue measure on $\mathbb{R}$. Since $\{|T(t)|\}_{t \in \mathbf{R}}$ is a positive $C_{0}$-group, it follows from [dP, 2.3] that $\forall \mu$ in $C_{0}(X)^{\odot d}|T(t)|^{*} \mu \perp \mu m$-a.e. on $\mathbb{R}$. It follows from [MN, 3.1.21] that both $T^{*}(t)$ and $|T(t)|^{*}$ are disjointness preserving, and therefore 
by [AB, 8.6] $\left|T^{*}(t) \mu\right|=\left|T^{*}(t)\right||\mu|$ and $|T(t)|^{*}|\mu|=\left.|| T(t)\right|^{*} \mu \mid$. Since by [MN, 3.1.21] $\left|T^{*}(t)\right|=|T(t)|^{*}$, we obtain:

$$
\left|T^{*}(t) \mu\right|=\left|T^{*}(t)\right||\mu|=|T(t)|^{*}|\mu|=\left.|| T(t)\right|^{*} \mu \mid .
$$

Therefore, $\left|T(t)^{*} \mu\right| \wedge|\mu|=\left.|| T(t)\right|^{*} \mu|\wedge| \mu \mid=0$ which implies that $T(t)^{*} \mu \perp \mu m$-a.e. on $\mathbb{R}$. The desired equality can now be obtained by mimicking the argument on p. 108 of $[\mathrm{dP}]$.

\section{ACKNOWLEDGMENTS}

The author wishes to express his gratitude to Professor W. A. J. Luxemburg for constant guidance during this project and to Dr. J. van Neerven for a number of useful discussions.

\section{REFERENCES}

[AB] C. D. Aliprantis and O. Burkinshaw, Positive operators, Academic Press, Orlando, FL, 1985. MR 87h: 47086

[dP] B. de Pagter, A Wiener-Young type theorems for dual semigroups, Positive Operators and Semigroups on Banach Lattices (C. B. Huijsmans and W. A. J. Luxemburg, eds.), Kluwer Academic Publisher, Dordrecht, The Netherlands, 1992, pp. 101-109. MR 93j:47059

[DS] N. Dunford and T. Schwartz, Linear operators, Interscience Publishers, Inc., New York, 1958. MR 22:8302

$[\mathrm{Ku}]$ M. Kuczma, Functional equations in a single variable, Polish Acad. Sci. Monograph in Math., vol. 46, Polish Acad. Sci., Warsaw, 1968. MR 37:4441

[LZ] W. A. J. Luxemburg and A. C. Zaanen, Riesz spaces I, North-Holland, Amsterdam, 1971. MR 58:23483

[MN] P. Meyer-Nieberg, Banach lattices, Springer-Verlag, Berlin, 1991. MR 93f:46025

[Na] R. Nagel (ed.), One-parameter semigroups of positive operators, Lecture Notes in Math., vol. 1184, Springer-Verlag, Berlin, 1984. MR 88i:47022

[Pa] A. Pazy, Semigroups of linear operators and applications to partial differential equations, Springer-Verlag, Berlin, 1983. MR 85g:47061

[Pl] A. Plessner, Eine Kennzeichnung der totalstetigen Funktionen, J. Reine Angew. Math. 60 (1929), 26-32.

$[\mathrm{Ru}]$ W. Rudin, Real and complex analysis, McGraw-Hill, New York, 1987. MR 88k:00002

[vN] J. van Neerven, The adjoint of a semigroup of linear operators, Springer-Verlag, Berlin, 1992. MR 94j:47059

[WY] N. Wiener and R. C. Young, The total variation of $g(x+h)-g(x)$, Trans. Amer. Math. Soc. 33 (1935), 327-340.

California Institute of Technology, 253-37, Caltech, Pasadena, California 91125

E-mail address: abiyanov@cco.caltech.edu

Current address: 155 Lexington St. \#33, Auburndale, MA 02166

E-mail address: biyanov@msn.com 